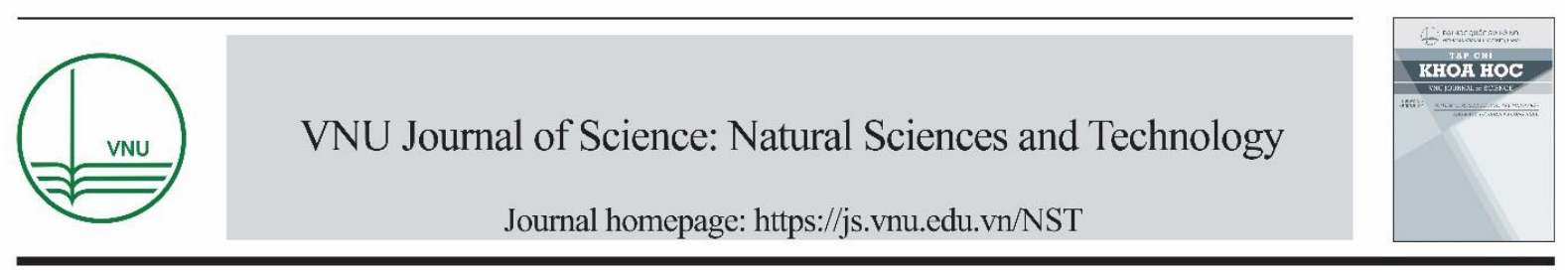

Original Article

\title{
Naringin Effectively Protects Cardiomyocytes Against Hypoxia/Reoxygenation Injury
}

\author{
Vu Thi Thu*, Ngo Thi Hai Yen \\ VNU University of Science, 334 Nguyen Trai, Thanh Xuan, Hanoi, Vietnam
}

Received 08 August 2021

Revised 19 August 2021; Accepted 20 August 2021

\begin{abstract}
This study was conducted to evaluate the protective effect of Naringin (NAR) on H9C2 cardiomyocytes in hypoxia/reoxygenation (HR) injury in vitro induced by the hypoxia chamber. In the study, H9C2 cells were grown under normal (control) and HR conditions. The viability, cardiolipin content and mitochondrial membrane potential of $\mathrm{H} 9 \mathrm{C} 2$ cells in the experimental group were analyzed by using suitable kits. The obtained results show that the addition of NAR $(16 \div 160 \mu \mathrm{M})$ significantly increased the survival rate of H9C2 cells under HR conditions. In particular, NAR showed remarkable efficiency in preserving mitochondrial function at concentrations of $80 \mu \mathrm{M}$ and $160 \mu \mathrm{M}$. In HR-exposed $\mathrm{H} 9 \mathrm{C} 2$ cell group, the cardiolipin content and mitochondrial membrane potential values of H9C2 cells decreased sharply $(71.64 \pm 1.37 \%$ and $68.12 \pm 2.78 \%$, $\mathrm{p}<0.05)$. Interestingly, mitochondrial cardiolipin contents signigicantly increased in $\mathrm{H} 9 \mathrm{C} 2$ cells post-hypoxic treated with NAR at doses of $80 \mu \mathrm{M}$ and $160 \mu \mathrm{M}$ to $87.76 \pm 1.89 \%$ and $81.09 \pm 1.21 \%$, respectively. Additionally, post-hypoxic supplementation of NAR at concentrations of $80 \mu \mathrm{M}$ and $160 \mu \mathrm{M}$ effectively increased mitochondrial membrane potential values. Overall, the obtained results are preliminary data on the effects of NAR in protecting mitochondria-targeted cardiomyocytes against HR injury.
\end{abstract}

Keywords: H9C2, cardiolipin, mitochondrial membrane potential.

\section{Introduction}

Hypoxia/reoxygenation (HR) injury is a myocardial tissue damage caused by revascularization to myocardial tissue after a period of time; this damage is ischemia or cessation of blood supply [1]. In fact, HR injury is a commonly used in vitro model of ischemia,

\footnotetext{
"Corresponding author.

E-mail address: vtthu2015@gmail.com

https://doi.org/10.25073/2588-1140/vnunst.5294
}

which is useful to study the recovery processes following the hypoxic period. Previous work has shown that mitochondria play important roles in the pathophysiology of HR cardiomyopathy [2]. Therefore, the preservation of mitochondrial function is necessary to limit damage to cardiomyocytes and myocardium. In the last few decades, the screening and finding of natural active substances with high biological activity, few side effects as well as optimized conditions to improve cardiac function in HR have had remarkable achievements [2, 3]. Of 
those, Naringin (NAR) is a flavanone glycoside found in high concentrations in the peels of citrus fruits (genus citrus) and has been shown to potent biological and pharmacological activities [4]. Previous research demonstrated that NAR possessed the anti-inflammatory and antioxidant activities [5]. Pretreatment with NAR attenuated renal and myocardial ischemia/reperfusion injury in rats $[6,7]$. Also, NAR pretreatment showed the potential to protect $\mathrm{H} 9 \mathrm{C} 2$ cells from HR-induced apoptosis [8]. However, the mechanism underlying the post-hypoxic treatement of NAR on $\mathrm{H} 9 \mathrm{C} 2$ cardiomyocytes remains poorly defined. Therefore, in this study, we investigated the effect of post-hypoxic treatment with NAR in HR-subjected $\mathrm{H} 9 \mathrm{C} 2$ by analyzing the cell viability as well as the mitochondrial structure and function.

\section{Materials and Methods}

\subsection{Materials}

The main materials and equipment used in this study were $\mathrm{H} 9 \mathrm{C} 2$ cell line (ATCC ${ }^{\circledR}$-USA), Naringin $\left(\mathrm{C}_{27} \mathrm{H}_{32} \mathrm{O}_{14} ;\right.$ Molecular weight: $580.53 \mathrm{~g} / \mathrm{mol} ; \geq 95 \%$ HPLC; National Institute of Medical Materials, Vietnam), Dulbecco's Modified Eagle Medium 4,5g/l glucose (DMEM, Gibco, USA), Fetal bovine serum (FBS, Gibco, USA), Penicillin-Streptomycin (PS, Gibco, USA), Phosphate buffered saline (PBS, Gibco, USA), Cell Counting Kit-8 (CCK-8, Dojindo, Japan), Dimethyl Sulfoxide (DMSO, Sigma, USA), Inverted microscopy Axiovert (S100, Carl Zeiss, Germany), Tetramethylrhodamine ethyl ester (TMRE, excitation/emission: 535/570 nm, Invitrogen, USA), 10-N-nonyl acridine orange (NAO, excitation/emission: 495/519 nm, Invitrogen, USA); Culture dishes 90x20 mm (SPL, Korea), 96-well black, glass bottom plates (CAT. 33196, SPL), confocal dishes (CAT. 100350, SPL), $\mathrm{CO}_{2}$ Incubator (Shellab, USA); ApoTome Fluorescence Microscope (Zeiss, Germany), and Microplate reader (Tristar, USA). The research was carried out at the Animal cell biotechnology laboratory, Life Science Research Center, Faculty of Biology, VNU University of Science.

\subsection{Methods}

\subsubsection{Cell Culture and Hypoxia-Reoxygenation} (HR) In Vitro Model

H9C2 cells were maintained culture dishes (90x20 nm) containing DMEM, 10\% FBS, and $100 \mu \mathrm{g} / \mathrm{ml}$ of PS at $37{ }^{\circ} \mathrm{C}$ with $5 \% \mathrm{CO}_{2}$. Culture medium was changed every 2-3 days. For HR in vitro model, $\mathrm{H} 9 \mathrm{C} 2$ cells were further transferred to either 96-well black plate, glass bottom or a confocal dish at density of $5.10^{3}$ cells/well at $37{ }^{\circ} \mathrm{C}, 5 \% \mathrm{CO}_{2}$. After $24 \mathrm{~h}$, the cells were then subjected to hypoxic condition and treatments as described in a previous publication [9] and the experimental cells were divided into different groups as follows: The normal control group: H9C2 cells were continously cultured under normal condition (DMEM, $10 \% \mathrm{FBS}, 1 \% \mathrm{PS}, 37^{\circ} \mathrm{C}$ and $5 \% \mathrm{CO}_{2}$ for $48 \mathrm{~h}$; the HR group: $\mathrm{H} 9 \mathrm{C} 2$ cells were cultured in serum-free low-glucose DMEM at $37{ }^{\circ} \mathrm{C}, 95 \% \mathrm{~N}_{2}, 5 \% \mathrm{CO}_{2}$, and $2 \% \mathrm{O}_{2}$ for $6 \mathrm{~h}$. Then, the old medium was removed and the H9C2 cells were then transferred to normal condition (DMEM, 10\% FBS, 1\% PS, $37{ }^{\circ} \mathrm{C}$ and $5 \% \mathrm{CO}_{2}$ ) for reoxygenation for $24 \mathrm{~h}$. At the time of reoxygenation (stimulating the reoxygenation stage), with the further treatments (i, ii):

i) HR group: the reoxygenation stage normal culture medium contained DMEM, $10 \%$ FBS, $1 \%$ PS;

ii) NAR groups: the reoxygenation stage medium contained DMEM, 10\% FBS, $1 \%$ PS, DMSO $0,1 \%$, and NAR at doses of 16, 32, 80, $160,320 \mu \mathrm{M}$ as previous study [10]. NAR stocks were prepared in DMSO and the final concentration of DMSO in cultured medium was about $0,1 \%$.

At the end of the experiment, the viability of cells was determined by CCK-8; cardiolipin content and mitochondrial membrane potential were measured indirectly through fluorescent indicators NAO and TMRE. 


\subsubsection{Measurement of Cell Viability}

The cell viability was determined by using CCK-8. After being subjected to HR and treatment, $\mathrm{H} 9 \mathrm{C} 2$ cell groups were further incubated for 1-4 $\mathrm{h}$ with CCK-8 as previously described [9]. For each group, an absorbance value indicating cell viability was measured at $450 \mathrm{~nm}$ using a microplate reader. The alive cell number in each well was expressed as a value relative to the normal control well. Experiments were repeated 4 times.

\subsubsection{Measurement of Mitochondrial} Cardiolipin and Mitochondrial Membrane Potential

Mitochondrial cardiolipin and mitochondrial membrane potential were indirectly assessed as in a previously discribed [9]. H9C2 cells were seeded in confocal dishes and subjected to HR model and treatments. After being subjected to different conditions, the cells were stained with either NAO $(0.1 \mu \mathrm{M}$, ex/em: $495 / 519 \mathrm{~nm})$ or TMRE ( $1 \mu \mathrm{M}$, ex/em: $535 / 570 \mathrm{~nm})$ at $37{ }^{\circ} \mathrm{C}$ for $30 \mathrm{~min}$ at room temperature to detect changes in cardiolipin content or mitochondrial membrane potential, respectively. After being washed with PBS, NAO- or TMRE-stained cells were captured using the ApoTome and the images were then reconstructed from individual tiles (X:6, Y:9) using ZEN Blue 2.5 software (Carl Zeiss). The total fluorescence intensities were expressed as percentage value relatives to the normal control. Experiments were performed for 3-4 times.

\subsubsection{Statistical Analysis}

Origin 8.5 software was chosen to analyse data. Data are presented as means \pm standard deviation (SD). Differences between the two groups were evaluated by ANOVA and Turkey test. A p-value $\leq 0.05$ was considered to be significant.

\section{Results and Discussion}

\subsection{NAR Exerts Weak Cytotoxic Effect on H9C2 Cells}

H9C2 cells were cultured in normal condition for $24 \mathrm{~h}$ and were then added NAR at dose of $16 \div 3200 \mu \mathrm{M}$ to culture media for $48 \mathrm{~h}$. The effects of NAR on the viability of H9C 2 cells assessed by using CCK- 8 are shown in Figure 1.

Previous research demostrated that NAR effects on cell viability in a dose-dependent manner [10, 11] and cell types [11, 12] via modulating different pathways. NAR inhibits proliferation and induces cell apoptosis of thyroid cancer cell [12]. NAR also protected H9C2 cells against damage caused by high glucose [10]. In consistence with previous study [10], the obtained results show that at concentration of $16 \div 3200 \mu \mathrm{M}$, NAR showed weak cytotoxic effect on $\mathrm{H} 9 \mathrm{C} 2$ cell with $\mathrm{IC}_{50}$ value of NAR was about 1,174.24 $\mu \mathrm{M}$ (Figure 1). We chose a concentration range of $16 \div 320 \mu \mathrm{M}$ to further investigate the effect of NAR on the viability of $\mathrm{H} 9 \mathrm{C} 2$ cells under HR conditions targeting mitochondrial function.

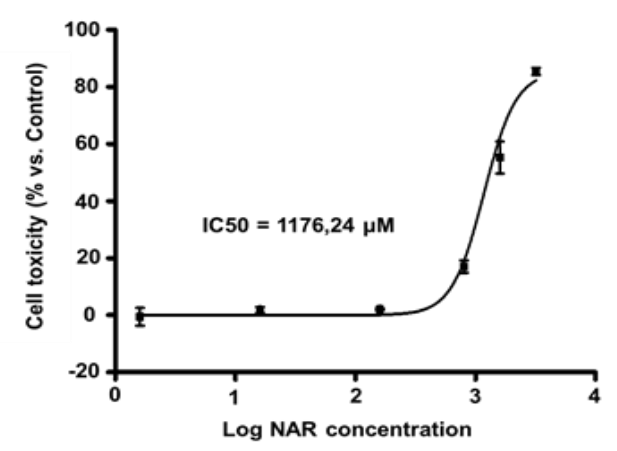

Figure 1 . The toxicity of Naringin on H9C2 cells. Naringin (NAR) concentration $(16 \div 3200 \mu \mathrm{M})$.

\subsection{NAR Reduces H9C2 Cell Death in HR Injury}

The effects of NAR at different concentrations on the viability of $\mathrm{H} 9 \mathrm{C} 2$ cells under HR conditions are presented in Figure 2 and Figure 3.

The representative image of $\mathrm{H} 9 \mathrm{C} 2$ cells shown in Figure 2 demonstrated that the number of dead cells in HR group (Figure 2B) is much higher than that in the normal control group (Figure 2A). Cell detachment, death and floating in the culture medium were clearly observed in HR-subjected cell group. Treatment 
with NAR increased the density of adhesive cells subjected to HR conditions (Figures 2C, D). The morphology result is quite similar to the $\mathrm{H} 9 \mathrm{C} 2$ cell viability result acquired by CCK-8 (Figure 3 ).
Under normal condition, NAR has no protective effects on $\mathrm{H} 9 \mathrm{C} 2$ cells (Figure $3 \mathrm{~A}$ ). However, it exerts strong protective effects in HR-stimulated conditions (Figure 3B).
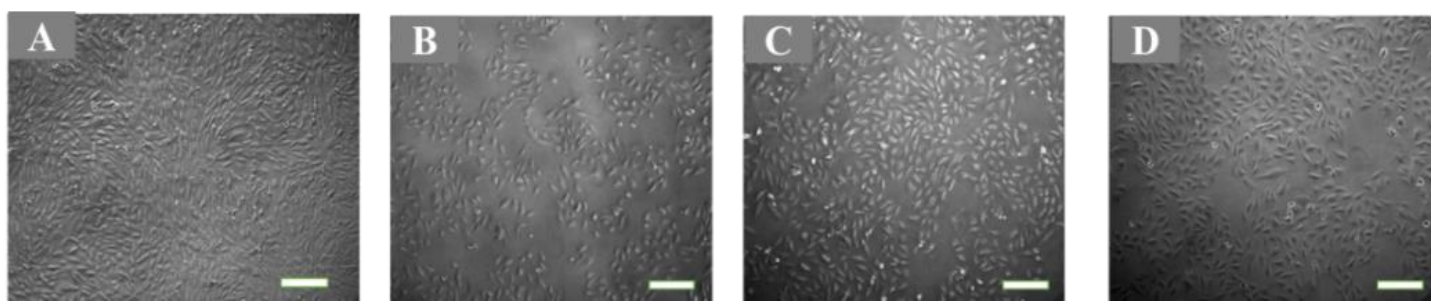

Figure 2. Images of H9C2 cells under different conditions.

A: H9C2 cells were cultured in normal condition; B: H9C2 cells were cultured in hypoxia/reoxygenation (HR) condition; C: H9C2 cells were cultured in HR condition and subjected to post-hypoxic treatment with $80 \mu \mathrm{M}$ NAR; D: H9C2 cells were cultured in HR condition and subjected to post-hypoxic treatment with $160 \mu \mathrm{M}$ NAR; $5 \mathrm{X}$ magnification; scale bar: $5 \mu \mathrm{m}$.
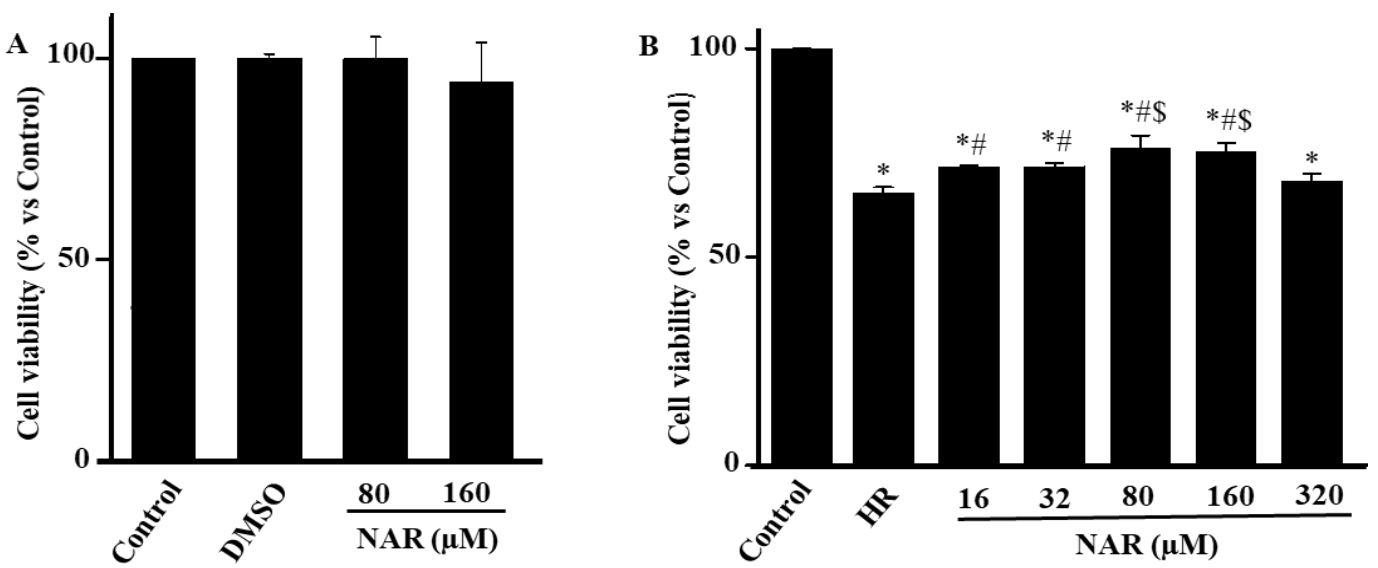

Figure 3. Cell viability of H9C2 under different conditions.

A: H9C2 cells cultured in normoxia condition; B: H9C2 cells subjected to hypoxia/reoxygenation (HR) model and treatment with Naringin (NAR). Control: H9C2 cells were cultured in normal condition (normoxia); DMSO: H9C2 cells were cultured in normoxia condition plus DMSO 0.1\%; HR: H9C2 cells were cultured in HR condition; NAR: H9C2 cells were cultured in normoxia or conditions of post-hypoxic treatment with NAR at doses of $16,32,80,160$ and $320 \mu \mathrm{M} ;{ }^{*} \mathrm{p}<0,05$ vs. control, ${ }^{*} \mathrm{p}<0,05$ vs. HR, ${ }^{\$} \mathrm{p}<0,05$ vs. NAR at dose of $320 \mu \mathrm{M} ; \mathrm{n}=4$.

The obtained results showed that survival rate in HR-exposed cells was significant lower $(65.50 \pm 3.75 \%)$ than those in control group $(\mathrm{p}<0.05$, Figure 3B). Post-hypoxic treatment with NAR at dose of $16 \div 160 \mu \mathrm{M}$ markedly increased the cellular survival rate compared to those in HR group $(\mathrm{p}<0.05)$. NAR treatments at doses of $80 \mu \mathrm{M}$ and $160 \mu \mathrm{M}$ showed highly cellular protective effect with cell alive percentage $(\%$ of control) were about $76.42 \pm 2.90$ and $75.32 \pm 2.25 \%$, respectively.
These results are quite similar to previous report [8]. Pretreatment with NAR increased the survival rate of $\mathrm{H} 9 \mathrm{C} 2$ cells againt HR injury through inhibition of oxidative free radical production. The effects of NAR resemble a NAR derivative, Naringenin. Previous studies proved that Naringenin was able to protect cells against HR injury [8, 13]. Also, Naringenin significantly reduced the cell viability of A431 cells with a concomitant increase in nuclear condensation and DNA fragmentation in a dose 
dependent manner [11]. The findings in this study further confirmed the effect of NAR on ischemic-reperfusion injury models in vivo $[5,14]$. In these researches, NAR treatment reduced infarct size compared to the non-treated group. Moreover, NAR was reported to show its protective role through inhibition of inflammatory response [15], oxidative stress [16], and preservation of mitochondrial structure [17]. Here, the cell viability in cell group treated with NAR at doses of $80 \mu \mathrm{M}$ and $160 \mu \mathrm{M}$ was not significantly different ( $\mathrm{p}>0.05$ ). Based on this result, we decided to choose NAR treatment at these two doses for further evaluation.

\subsection{Naringin Ameliorates Mitochondrial Dysfunction in HR-subjected H9C2 Cells}

Mitochondria are powerhouse of cells and play important roles in cellular biological process. In this study, the effects of NAR on mitochondria of $\mathrm{H} 9 \mathrm{C} 2$ cells were assessed via mitochondrial cardiolipin contents and mitochondrial membrane potential indexes.

\subsubsection{Naringin Preserves the Mitochondrial Cardiolipin Content}

Cardiolipin is a characteristic phospholipid of the mitochondrial inner membrane and plays an important role in the structure and functioning of the respiratory chain [18]. Cardiolipin was reported to be involved in the apoptosis of animal cells through interaction with several lethal proteins [19]. In HR pathophysiology, decrease in content impaired electron transport chain activity, subsequently disrupting mitochondrial function and leading to cell death [20]. Therefore, maintaining stable levels of cardiolipin is important to limit mitochondrial and cellular damage in cardiomyopathy [21]. In this study, the changes in cardiolipin content among cell groups were determined by using NAO fluorescence assay (Figure 4).
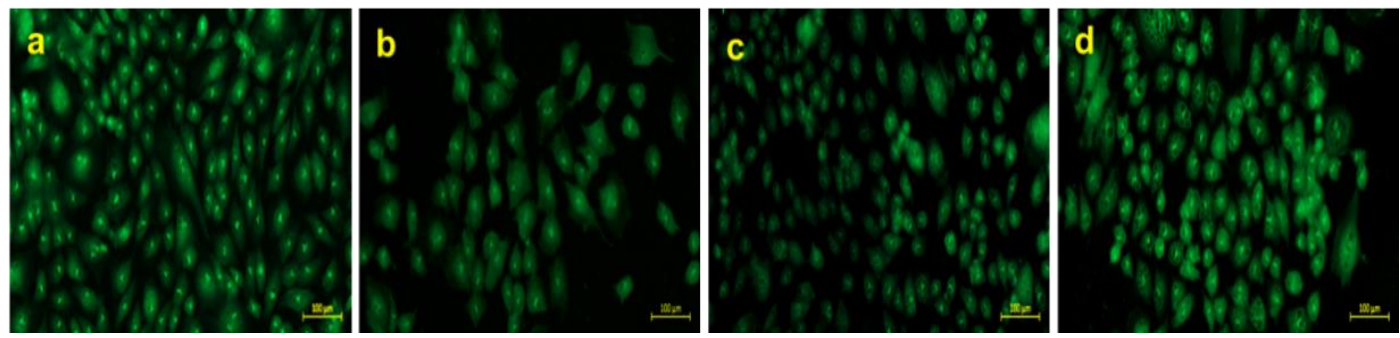

A

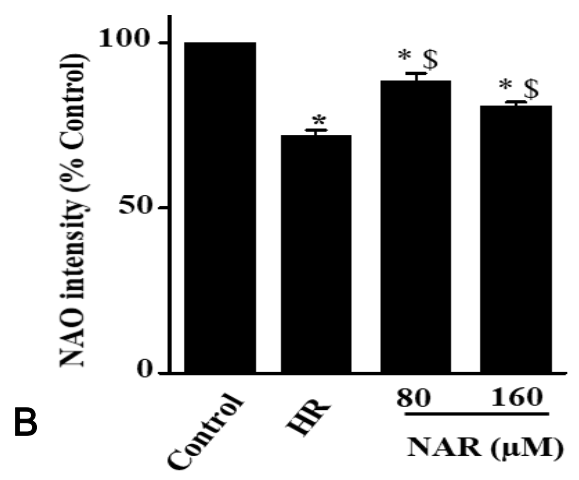

Figure 4. NAO intensity in $\mathrm{H} 9 \mathrm{C} 2$ cells.

A: Representative images of NAO-stained H9C2 cells under normal control (a), hypoxia/reoxygenation (HR, b), post-hypoxic treatment with Naringin (NAR) at dose of $80 \mu \mathrm{M}$ (c), $160 \mu \mathrm{M}$ (d) conditions; B: NAO intensity in different conditions. ${ }^{*} \mathrm{p}<0.05$ vs. control, ${ }^{\$} \mathrm{p}<0.05$ vs. HR, scale bar $=100 \mu \mathrm{m}, \mathrm{n}=4$. 
The results in Figure 4 show that HR group has the lowest NAO intensity, remaining only about $71.64 \pm 1.37 \%$ of control. Interestingly, NAO fluorescence intensities of cell groups treated with NAR at doses of $80 \mu \mathrm{M}$ and $160 \mu \mathrm{M}$ were highly increased and were about $87.76 \pm 1.89$ and $81.09 \pm 1.21 \%$ (of $100 \%$ control), respectively. This shows that NAR at concentrations of 80 and $160 \mu \mathrm{M}$ has the ability to preserve mitochondrial cardiolipin in HR-induced injury; thereby, increasing the efficiency of the electron transport chain. This was consistent with previous study [20]. Besides, the data in Figure 4 also show that post-hypoxic treatment with NAR at dose of $80 \mu \mathrm{M}$ had higher value of the NAO fluorescence intensity than those in cell group treated with NAR at concentration of $160 \mu \mathrm{M}$. However, this difference was not statistically significant ( $>>0.05$ ). This result is quite consistent with the change in survival of H9C 2 cells (Figure 3 ).

\subsubsection{Naringin Preserves Mitochondrial Membrane Potential}

Mitochondrial membrane potential is an important parameter for assessing mitochondrial function. The collapse of membrane potential might lead to mitochondrial malfunction and cell death [22]. In this study, mitochondrial membrane potential index was indirectly assessed by measuring TMRE fluorescence intensity (Figure 5).
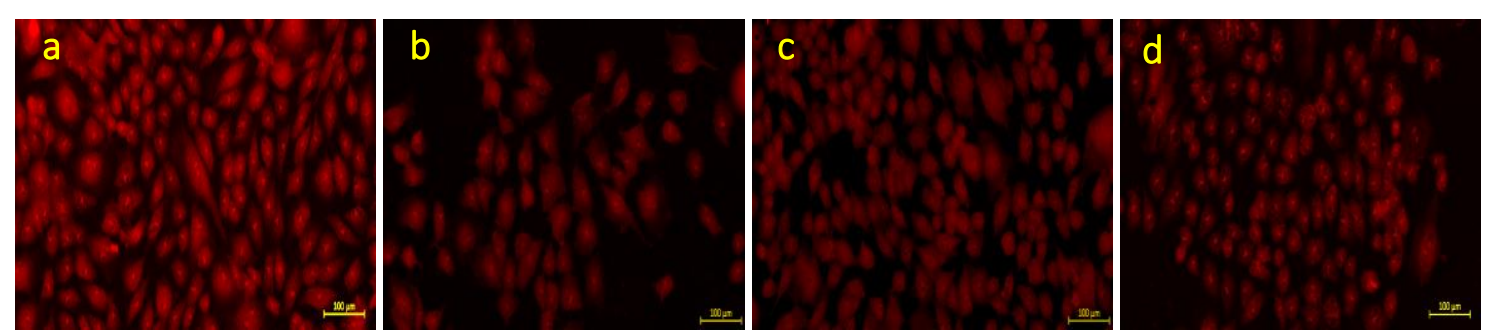

A

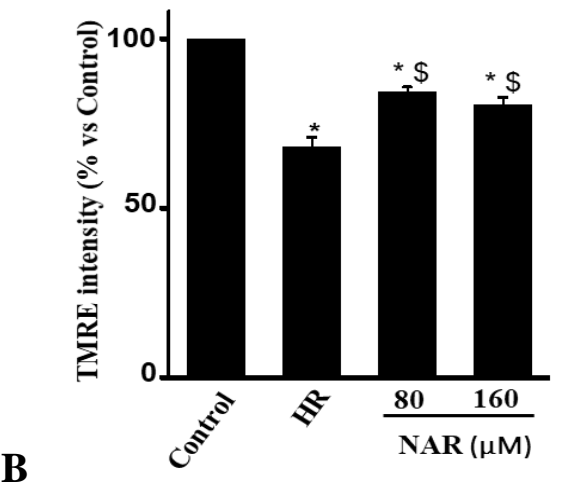

Figure 5. TMRE intensity in $\mathrm{H} 9 \mathrm{C} 2$ cells.

A: Representative images of TMRE-stained H9C2 cells under normal control (a), hypoxia/reoxygenation (HR, b), post-hypoxic treatment with Naringin (NAR) at dose of $80 \mu \mathrm{M}$ (c), $160 \mu \mathrm{M}$ (d) conditions; B: TMRE intensity in different conditions. ${ }^{*} \mathrm{p}<0.05$ vs. control, ${ }^{\$} \mathrm{p}<0.05$ vs. HR, scale bar $=100 \mu \mathrm{m}, \mathrm{n}=3 \div 4$.

Figure 5A shows the representative images of TMRE-stained H9C2 cells captured in different groups. In this assay, the redder density shows the higher mitochondrial membrane potential. The results show that HR sharply reduced mitochondrial membrane potential, therefore markedly inducing mitochondrial malfunction. In HR group, the number of red cells is smaller than those in other groups (Figure 5A). Percentage of TMRE fluorescence intensity in HR was $68.12 \pm 2.78$ (of $100 \%$ control, Figure 5B). The cell groups 
supplemented with NAR at concentrations of $80 \mu \mathrm{M}$ and $160 \mu \mathrm{M}$ had the brighter red signal as well as a higher TMRE fluorescence intensity $(84.32 \pm 1.49 \%$ and $80.60 \pm 2.19 \%$ respectively) compared to those in HR group $(\mathrm{p}<0.05)$. The results suggest the ability of NAR in preserving mitochondrial membrane potential under HR condition. It is reported that stabilizing mitochondrial membrane potential reduces mitochondrial dysfunction and increases cell viability [23]. This result is quite consistent with last study [8]. Pretreatment with NAR at concentration of $10-40 \mu \mathrm{g} / \mathrm{ml}$ might preserve the mitochondrial membrane potential against HR, thereby limiting programmed cell death. The mitochondrial membrane potential value is relatively consistent with both the cell survival rate (Figure 2, 3) and the mitochondrial membrane cardiolipin content (Figure 4). Interestingly, post-hypoxic treatment of NAR at doses $80 \mu \mathrm{M}$ and $160 \mu \mathrm{M}$ had no significant difference. The results indicate that the most effective dose of NAR against HR damage was approximately $80 \mu \mathrm{M}$.

Thus, the results show that NAR has the ability to protect $\mathrm{H} 9 \mathrm{C} 2$ rat cardiomyocytes through targeting mitochondria. However, the detail cytoprotective mechanism of NAR on HR-related molecules is still unknown and should be elucidated in further study.

\section{Conclusion}

The study documented that post-hypoxic treatment with NAR significantly increased the viability of disease-modified H9C2 cells through preserving mitochondrial structure and funtion. The new role of NAR may be potential for attenuating HR damage.

\section{Acknowledgements}

The authors thank Dr. Nguyen Ha Ly for providing Naringin; and Dr. Pham Thi Bich, BSc. Nguyen Phuong Thao, BSc. Ho Ly Phuong, BSc. Nguyen Thi Thuy Hang, Assoc. Prof. Nguyen Lai Thanh, and Prof. Han Jin for help and support.
This work was supported by the National Foundation for Science and Technology (NAFOSTED, 106-YS.06-2016.23).

\section{References}

[1] T. Kalogeris et al., Ischemia/Reperfusion, Comprehensive Physiology, Vol. 7, No. 1, 2016, pp. 113-170, https://doi.org/10.1002/ cphy.c160006.

[2] A. Heller, G. Brockhoff, A. Goepferich, Targeting Drugs to Mitochondria, European Journal of Pharmaceutics and Biopharmaceutics, Vol. 82, No. 1, 2012, pp. 1-18, https://doi.org/10.1016/j.ejpb.2012.05.014.

[3] M. Andrew, M. A. George, S. Paul, Mitochondria as a Drug Target in Ischemic Heart Disease and Cardiomyopathy, Circulation Research, Vol. 111, No. 9, 2012, pp. 1222-1236, https://doi.org/10.1161/CIRCRESAHA.112.265660.

[4] M. A. Alam et al., Effect of Citrus Flavonoids, Naringin and Naringenin, on Metabolic Syndrome and Their Mechanisms of Action, Advances in Nutrition (Bethesda, Md.), Vol. 5, No. 4, 2014, pp. 404-417, https://doi.org/10.3945/an.113.005603.

[5] A. Cerkezkayabekir et al., Naringin Protects Viscera from Ischemia/reperfusion Injury by Regulating the Nitric Oxide Level in a Rat Model, Biotech Histochem, Vol. 92, No. 4, 2017, pp. 252-263, https://doi.org/10.1080/10520295.2017.1305499.

[6] D. Singh, K. Chopra, The Effect of Naringin, a Bioflavonoid on Ischemia-reperfusion Induced Renal Injury in Rats, Pharmacological Research, Vol. 50, No. 2, 2004, pp. 187-193, https://doi.org/10.1016/j.phrs. 2004.01.007.

[7] F. Li et al., Naringin Attenuates Rat Myocardial Ischemia/reperfusion Injury Via PI3K/Akt Pathway-mediated Inhibition of Apoptosis, Oxidative Stress and Autophagy, Experimental and Therapeutic Medicine, Vol. 22, No. 2, 2021, pp. 811, https://doi.org/ 10.3892/etm.2021.10243.

[8] R C. Chen et al., Naringin Protects Against Anoxia/reoxygenation-induced Apoptosis in H9c2 Cells Via the Nrf2 Signaling Pathway, Food and Function, Vol. 6, No. 4, 2015, pp. 1331-44, https://doi.org/10.1039/c4fo01164c.

[9] V. T. Thu et al., Majonoside-R2 Extracted from Vietnamese Ginseng Protects H9C2 Cells Against Hypoxia/reoxygenation Injury Via Modulating Mitochondrial Function and Biogenesis, Bioorganic and Medicinal Chemistry Letters, Vol. 36, 2021, pp. 127814, https://doi.org/10.1016/j.bmcl.2021.127814. 
[10] J. Chen et al., Naringin Inhibits ROS-activated MAPK Pathway in High Glucose-induced Injuries in H9C2 Cardiac Cells, Basic \& Clinical Pharmacology and Toxicology, Vol. 114, No. 4, 2014, pp. 293-304, https://doi.org/10.1111/bcpt.12153.

[11] M. S. Ahamad et al., Induction of Apoptosis and Antiproliferative Activity of Naringenin in Human Epidermoid Carcinoma Cell through ROS Generation and Cell Cycle Arrest, PLOS ONE, Vol. 9, No. 10, 2014, pp. e110003, https://doi.org/10.1371/journal.pone.0110003.

[12] J. Zhou, L. Xia, Y. Zhang, Naringin Inhibits Thyroid Cancer Cell Proliferation and Induces Cell Apoptosis Through Repressing PI3K/AKT Pathway, Pathology Research and Practice, Vol. 215, No. 12, 2019, pp. 152707, https://doi.org/10.1016/j.prp. 2019.152707.

[13] J. Y. Tang et al., Naringenin Ameliorates Hypoxia/reoxygenation-induced Endoplasmic Reticulum Stress-mediated Apoptosis in $\mathrm{H} 9 \mathrm{c} 2$ Myocardial Cells: Involvement in ATF6, IRE1 $\alpha$ and PERK Signaling Activation, Molecular and Cellular Biochemistry, Vol. 424, No. 1, 2017, pp. 111-122, https://doi.org/10.1007/s11010-016-2848-1.

[14] J. Feng et al., Naringin Attenuates Cerebral Ischemia-Reperfusion Injury Through Inhibiting Peroxynitrite-mediated Mitophagy Activation, Mol Neurobiol, Vol. 55, No. 12, 2018, pp. 9029-9042, https://doi.org/10.1007/s12035-018-1027-7.

[15] L. J. Sun, et al., Naringin Mitigates Myocardial Strain and the Inflammatory Response inSepsis-induced Myocardial Dysfunction through Regulation of $\mathrm{PI} 3 \mathrm{~K} / \mathrm{AKT} / \mathrm{NF}-\mathrm{\kappa B} \quad$ Pathway, International Immunopharmacology, Vol. 75, 2019, pp. 105782, https://doi.org/10.1016/ j.intimp.2019.105782.

[16] M. C. Saiz et al., Antioxidant Properties, Radical Scavenging Activity and Biomolecule Protection Capacity of Flavonoid Naringenin and Its Glycoside Naringin: A Comparative Study, The Journal of the
Science of Food and Agriculture, Vol. 90, No. 7, 2010, pp. 1238-1244, https://doi.org/10.1002/jsfa.3959.

[17] L. J. Sun et al., Layer-specific Strain for Assessing the Effect of Naringin on Systolic Myocardial Dysfunction Induced by Sepsis and Its Underlying Mechanisms, The Journal of International Medical Research, Vol. 49, No. 1, 2021, pp. 300060520986369 , https://doi.org/10.1177/0300060520986369.

[18] J. Dudek, Role of Cardiolipin in Mitochondrial Signaling Pathways. Frontiers in Cell and Developmental Biology, Vol. 5, No. 90, 2017, https://doi.org/10.3389/fcell.2017.00090.

[19] G. Paradies et al., Cardiolipin Alterations and Mitochondrial Dysfunction in Heart Ischemia/reperfusion Injury, Clinical Lipidology, Vol. 10, No. 5, 2015, pp. 415-429, https://doi.org/10.2217/clp.15.31.

[20] G. Paradies et al., Mitochondrial Bioenergetics and Cardiolipin Alterations in Myocardial Ischemia-reperfusion Injury: Implications for Pharmacological Cardioprotection, The American Journal of Physiology-Heart and Circulatory Physiology, Vol. 315, No. 5, 2018, pp. H1341-H1352, https://doi.org/10.1152/ajpheart.00028.2018.

[21] U. Schlattner et al., Mitochondrial Kinases and Their Molecular Interaction with Cardiolipin, Biochimica et Biophysica Acta (BBA) - Biomembranes, Vol. 1788, No. 10, 2009, pp. 2032-2047, https://doi.org/10.1016/j.bbamem.2009.04.018.

[22] M. N. Sack, Mitochondrial Depolarization and the Role of Uncoupling Proteins in Ischemia Tolerance, Cardiovascular Research, Vol. 72, No. 2, 2006, pp. 210-219, https://doi.org/10.1016/ j.cardiores.2006.07.010.

[23] L. D. Zorova et al., Mitochondrial Membrane Potential, Analytical Biochemistry, Vol. 552, 2018, pp. 50-59, https://doi.org/10.1016/j.ab. 2017.07.009. 